\title{
Bases teóricas del uso simulación para el entrenamiento en cirugía
}

\author{
Juan Enrique Berner ${ }^{1,2}$ y Ernesto Ewertz ${ }^{3}$
}

${ }^{1}$ Kellogg College, University of
Oxford, Oxford, Reino Unido.
2Queen Victoria Hospital,
Departamento de Cirugía
Plástica, East Grinstead, Reino
Unido.
3Hospital del Trabajador,
Santiago, Chile.
Recibido el 14 de noviembre
de 2017 y aceptado para
publicación el 20 de diciembre
de 2017.

Correspondencia a: Dr. Ernest Ewertz efewertz@gmail.com

\section{Theoretical bases of the use of simulation for training in surgery}

In the current healthcare scenario, several factors have been identified as threats for medical education. The increase in litigations, working-time directives, patient safety issues and a higher social demand for specialists have forced medical educators to look for new alternatives to traditional teaching. Simulation provides a recreation of a clinical situation, allowing the student to acquire skills and competences that then can be translated to real life practice. This article summarises the key concepts of simulation, the educational theories that back up its use, its limitations and the available evidence in regards to its use.

Key words: simulation; medical training; surgery.

\section{Resumen}

En el contexto clínico actual, diversos factores entorpecen la formación de médicos y especialistas. La judicialización de la práctica médica, legislaciones que limitan las horas trabajadas, una cultura de trabajo que no admite errores y una mayor demanda por especialistas son desafíos que han obligado a docentes a buscar nuevas herramientas. La simulación permite recrear una situación clínica, permitiendo al alumno adquirir competencias previo a su aplicación en la vida real. Este trabajo resume los conceptos actuales en simulación, las bases teóricas educacionales que explican su utilidad, sus limitaciones y la evidencia disponible sobre su utilización.

Palabras clave: simulación; educación médica; cirugía.

\section{Introducción}

Los resultados de un siglo de innovación en educación médica son evidentes desde donde nos encontramos. Junto con los avances en salud pública y mejoras en el acceso a la atención de salud, los profesionales entrenados durante el siglo $\mathrm{XX}$, fueron los responsables de duplicar la expectativa de vida de países desarrollados y en vías de desarrollo. Sin embargo, un contexto cambiante significa desafíos cambiantes para la disciplina de la educación médica.

Avances sin precedentes en las tecnologías de la información y telecomunicaciones en las últimas décadas han cambiado la forma en que se desarrolla prácticamente toda forma de actividad humana, incluyendo la relación médico-paciente ${ }^{1,2}$. En res- puesta a esta situación ha surgido una conversión hacia un modelo de cuidado de salud centrado en el paciente, reemplazando el modelo puramente biomédico enfocado en la enfermedad. Consecuentemente, el principio Hipocrático de primum non nocere ha tomado revitalizada preponderancia en las últimas dos décadas. Comenzando con el reporte $T o$ Err is Human, publicado por el Instituto Americano de Medicina en 1999, la seguridad del paciente se ha convertido en la piedra angular de cualquier intervención médica, permeando a los currículo de pre y posgrado ${ }^{3}$. Se estima que los errores médicos prevenibles resultan en más de 400.000 muertes al año en EE. UU., ubicándose como la tercera causa de muerte en este país y la iatrogenia no fatal genera incapacidad en más de 3,5 millones de pacientes anualmente. 
Por su parte, la judicialización de la práctica médica y el empoderamiento del paciente con respecto al conocimiento de su patología ha derivado en un escenario donde simplemente el error no es aceptable. Solo en Estados Unidos, durante la década consistente entre el 2000 y 2010 , en promedio 4.850 causas legales por negligencia médica resultaron en algún tipo de compensación financiera ${ }^{4}$.

Inevitablemente el fenómeno ha impactado en la formación de especialistas, especialmente en aquellas ramas involucradas en procedimientos invasivos. Se ha reportado una tendencia a restringir las oportunidades educacionales, en la premisa que un especialista completamente formado es más seguro que uno en formación. Consecuentemente, entrenar especialistas competentes se convierte en una tarea difícil y un desafío que obliga a replantear los principios de educación médica basado en el aprendizaje en los propios pacientes ${ }^{5}$.

Por otro lado, no es solo el contexto social el que afecta el entrenamiento quirúrgico de postgrado hoy en día, sino también directivas legislativas introducidas para limitar el número de horas que los residentes debieran trabajar tanto en Norteamérica como en Europa ${ }^{6}$. Particularmente, en el Reino Unido, la implementación de la Directiva Europea de Tiempo Trabajado ha sido sujeto de un debate en cuanto al impacto que ha tenido en la formación de especialistas, especialmente en lo que respecta a las competencias prácticas ${ }^{7}$. Un reciente artículo concerniendo a la formación de cirujanos plásticos en Inglaterra demostró que, a pesar de que los residentes reconocen una mejoría en su calidad de vida, consecuentemente sus registros operatorios mostraron una reducción en un $30 \%$ en el número de casos en los que participaron ${ }^{8}$.

En este contexto ${ }^{9}$ ¿Cómo logramos entrenar nuevos especialistas competentes sin exponer a los pacientes a posibles consecuencias de su inexperiencia? ¿Cómo ponderamos la necesidad de adquisición de destrezas y conocimientos prácticos de los residentes con la seguridad que requiere cualquier procedimiento al intervenir sobre un ser humano? Si buscamos la respuesta a estas interrogantes en otras disciplinas encontramos la respuesta en la simulación, como ya lo ha hecho previamente la industria aeronáutica, aeroespacial y militar entre otras. En estas el entrenamiento basado en la simulación es parte fundamental de la malla curricular, lo que ha permitido entrenar y estandarizar conductas frente a situaciones habituales y a exponer a los sujetos en formación a situaciones poco frecuentes, pero de elevado riesgo que en otras condiciones no podrían ser experimentadas.

\section{Simulación como herramienta docente}

Un simulador es un sistema artificial capaz de replicar aspectos de una situación clínica específica, dándole la oportunidad al alumno de practicar habilidades psicomotoras, técnicas y de juicio clínico ${ }^{10,12}$. Provee un ambiento seguro, donde la repetición es recomendada, para que el alumno aprenda de sus propios errores, sin las consecuencias que normalmente tendrían en su ambiente laboral. A pesar, que la utilización de simuladores para la docencia clínica data de más de 2.500 años, particular atención se empieza a prestar a ella durante la década de 1960 con el desarrollo de los modelos Resusci Anne y SimOne, enfocados en el entrenamiento en resucitación en residentes de anestesia ${ }^{13}$. Los progresivos avances en las tecnologías de la simulación han llevado a que la simulación hoy en día no sea un elemento extraño en la formación de cualquier especialista.

Un simulador intenta replicar una situación clínica para proveer una instancia educativa para el estudiante. La suposición básica recae en que, si el simulador imita de manera fidedigna la realidad, las lecciones y habilidades aprendidas podrán ser aplicables en la clínica. En este sentido, el concepto de fidelidad es útil para describir hasta qué nivel la apariencia y comportamiento del simulador replica la apariencia y comportamiento del sistema real ${ }^{14}$.

Algunos autores han reducido el concepto de fidelidad a una mera descripción del nivel de complejidad tecnológica del simulador, distinguiendo entre alta-fidelidad y baja-fidelidad basándose en especificaciones técnicas ${ }^{15}$. Una manera más comprensiva de entender el concepto de fidelidad recae en no solo considerar el dispositivo en sí, sino la calidad de la interacción entre simulador y estudiante. La experiencia simulada no solo imita características del mundo físico, pero también en cierto grado imita una tarea que despierta una cadena de pensamientos, acciones y hasta emociones en el alumno. Más aún, el simulador también puede reproducir claves visuales y sensoriales en el ambiente que rodea la tarea en cuestión, acercándose detalladamente a la experiencia real. Considerando estas tres dimensiones es que la fidelidad puede ser clasificada en: física, psicológica y ambiental ${ }^{16}$.

El nivel de fidelidad requerido para un simulador específico dependerá entonces de la situación clínica específica y del aprendizaje esperado. Replicar cualquier escenario al mayor nivel de fidelidad puede ser no solo costoso, pero también poco práctico y posiblemente poco ético. Para algunas tareas, un simulador de baja fidelidad puede ser la 
mejor opción. Los simuladores de punción venosa y canulación son un ejemplo clásico, consistiendo en un brazo de plástico con una serie de tubos llenos de un fluido rojo para que estudiantes de medicina practiquen esta competencia, previo a realizarlo en pacientes. En sí mismo es un simulador que difiere considerablemente de la situación real, no solo porque el paciente está reducido a un brazo de plástico insensible pero también en que el plástico se siente muy distinto a la piel y venas reales. Sin embargo, este part-task trainer ha probado su utilidad, en que el estudiante puede practicar la serie de pasos necesarios para hacer la tarea de manera segura e incluso puede, con un poco de imaginación, practicar habilidades comunicacionales.

De todos modos, algunos otros escenarios clínicos pueden requerir de mayor fidelidad. La anastomosis vascular microquirúrgica es una habilidad compleja que requiere entrenamiento previo a su práctica clínica. Una pulida técnica es necesaria para que el estudiante sea considerado un operador seguro, principalmente porque un mínimo error puede poner en riesgo una compleja y tediosa cirugía, tal como un colgajo libre o un reimplante digital. De manera de hallar una respuesta, docentes han encontrado en la simulación una solución, desarrollando distintos modelos para entrenar en microcirugía, con distintos niveles de fidelidad: desde aplicaciones en un teléfono celular, pasando por suturar un guante bajo el microscopio, anastomosar vasos sanguíneos en extremidades de aves hasta el modelo de arteria femoral en ratas vivas ${ }^{17}$. Uno de los aspectos más interesantes de todas estas alternativas es que se adaptan a la disponibilidad de recursos y a las necesidades de cada estudiante dependiendo del punto de la curva de aprendizaje en la que se encuentre. Se puede perfectamente comenzar con un simulador de baja fidelidad terminando en un modelo más complejo cuando las competencias son adquiridas.

\section{Bases teóricas educaciones de la simulación}

Desde una perspectiva conductista, la simulación permite al estudiante aprender de la experiencia y la repetición, adquiriendo las habilidades requeridas para la práctica clínica. Este nuevo repertorio de conductas puede después ser reforzadas o corregidas por los comentarios del docente durante la sesión. Desde esta teoría educacional la simulación sería particularmente útil para el aprendizaje de respuestas protocolizadas frente a estímulos concretos, como lo son los cursos de resucitación. En ellos los estudiantes son motivados a sobre-aprender una habilidad de manera que resulte automática a la hora de necesitarla.

Una visión diferente es la propuesta por la escuela cognitivista, quienes proponen que el conocimiento es adquirido mediante la interacción entre el estudiante, el simulador y el tutor, no solo mediante la realización de una tarea. Es el proceso cognitivo, en el cual el alumno confronta la nueva experiencia con sus ideas preexistentes, el responsable de la asimilación del conocimiento en la medida que se resuelve el conflicto cognitivo entre expectativas y realidad. De esta manera el estudiante se mueve desde los niveles más básicos de comprensión hacia los niveles superiores que según la taxonomía cognitiva de Bloom, comprenden la aplicación, análisis, síntesis y evaluación de lo aprendido ${ }^{18}$. El tutor juega un rol fundamental facilitando la clarificación de los conceptos, haciendo preguntas y guiando a lo largo del proceso cognitivo.

En cambio, según la teoría constructivista, esta interacción entre alumno y docente es considerada como componente fundamental. Mientras que el simulador provee la situación, es el tutor quien es capaz de dirigir al estudiante hacia el siguiente nivel de competencia, el cual sería difícil para el estudiante alcanzar de manera independiente. El objetivo es llevar al alumno a través de lo que Lev Vigotsky denominó Zona de Desenvolvimiento Proximal de una manera segura en un ambiente simulado, progresando hasta que sea capaz de ejecutarla de manera independiente en la práctica clínica ${ }^{14}$. Jerome Bruner en sus trabajos llamó este proceso un "andamiaje", por ser una estructura temporal que permite la consolidación de una más permanente.

Otro aspecto interesante de la simulación es que permite, tal cual como en la vida real, que el estudiante reflexione sobre su progreso. La posibilidad de reflexionar en acción (reflection-in-action) durante la simulación permite al estudiante en un instante adaptarse a la situación para lograr el resultado esperado, lo cual puede ser limitado conceptualmente, pero rico en detalles prácticos para completar una tarea. La más formal reflexión sobre la acción (reflection-on-action) puede venir más tarde para analizar el desenvolvimiento, emociones $\mathrm{y}$ resultados finales para lograr obtener conclusiones $\operatorname{abstractas}^{19}$.

A su vez la simulación provee una oportunidad sin par en el ámbito médico para la "práctica deliberada". De acuerdo con Ericsson y su teoría de adquisición de habilidades, para lograr un nivel superior de maestría y convertirse en un experto el aprendiz necesita comprometerse en repetición activa y esforzada, enfocándose en mejorar sus errores y 
buscando retroalimentación adecuada de manera de evitar lograr automaticidad en un estándar promedio, sino que apuntando a sobrepasar ese estándar ${ }^{20}$. Desafortunadamente esto toma tiempo, tal como Malcolm Gladwell ejemplifica en su libro Outliers ${ }^{21}$ mencionando las $10.000 \mathrm{~h}$ de práctica necesarias, lo cual no es particularmente placentero. La simulación sería entonces una herramienta fundamental para quienes deseen convertirse en expertos de la manera que lo define Ericsson.

Considerando las incontables posibilidades que la simulación otorga, la última década ha presenciado el desarrollo de nuevos y más complejos simuladores, usando las tecnologías de información disponibles actualmente. Los simuladores de baja fidelidad como el previamente mencionado simulador de venopunción fueron solo el puntapié para una nueva generación de simuladores generados por computadoras, realidad virtual y sistemas de simuladores hápticos, los cuales son capaces de imitar la fisiología completa de un paciente, e incluso simuladores ambientales para replicar instalaciones como pabellones o unidades de cuidados intensivos.

Las aplicaciones son ilimitadas y no solo para entregar contenidos, sino también como medios de evaluación del entrenamiento otorgado. La pirámide de Miller's es útil para ilustrar la utilidad de la simulación en la evaluación de competencias adquiridas $^{22}$ (Figura 1). Los dos primeros niveles de esta pirámide son puramente cognitivos: "saber" y "saber cómo", lo cual refleja la importancia inicial de la adquisición e interpretación de información. El siguiente paso es "demostrar cómo" en la cual el estudiante puede demostrar lo que ha aprendido hasta el momento. En el contexto en que las evaluaciones tienen que ser justas, objetivas y reproducibles para una cohorte de estudiantes, la vida real resulta ser impráctica. Mientras que la simulación en sí misma y embebida en Exámenes Controlados Objetivos Estandarizados (ECOEs) parecieran ser la herramienta ideal para medir que tan competente es un estudiante previo a que alcance el último nivel de la pirámide: "hace", lo cual es esperado en el ambiente laboral. Los pacientes simulados son particularmente útiles para enseñar y evaluar un dominio de habilidades difíciles de medir de otra manera como las habilidades comunicacionales, profesionalismo y aquellas no invasivas como el examen físico.

Por otro lado, la simulación no sólo permite el entrenamiento de especialistas en formación, sino además permite la planificación preoperatoria de pacientes con patologías complejas para cirujanos entrenados. Los avances en otras materias como la impresión $3 \mathrm{D}$ permiten en algunas materias practi- car la intervención a realizar, con las complejidades anatómicas particulares del paciente en cuestión, lo que permite anticiparse a situaciones inesperadas, prevenir errores y a optimizar tiempos quirúrgicos $\mathrm{y}$ resultados del procedimiento de forma segura y reproducible.

\section{Limitaciones y evidencia disponible}

La transferencia de habilidades desde el simulador hacia la vida real ha sido objeto de debate, porque ni el más complejo simulador es capaz de imitar ciento por ciento la imprevisibilidad, complejidad y sensibilidades que la vida real posee ${ }^{14}$. Mientras que un error en un simulador es una experiencia de aprendizaje, un error similar durante un procedimiento invasivo puede ser catastrófico para el paciente. La consecuente carga emocional que la práctica clínica tiene es imposible de reproducirse con un simulador. De la misma forma, la profundidad que una reflexión que un incidente o complicación real desencadena en un residente es una enseñanza difícil de borrar que un simulador no puede alcanzar.

También se ha recalcado que existe un riesgo que la simulación podría otorgar una sensación de falsa autoconfianza a ciertos residentes que no necesariamente hayan alcanzado las competencias necesarias para una tarea ${ }^{23}$, lo cual podría significar un riesgo para los pacientes. El rol del supervisor es fundamental de manera de temperar esta percepción en los estudiantes, enfatizando en las limitaciones de la simulación.

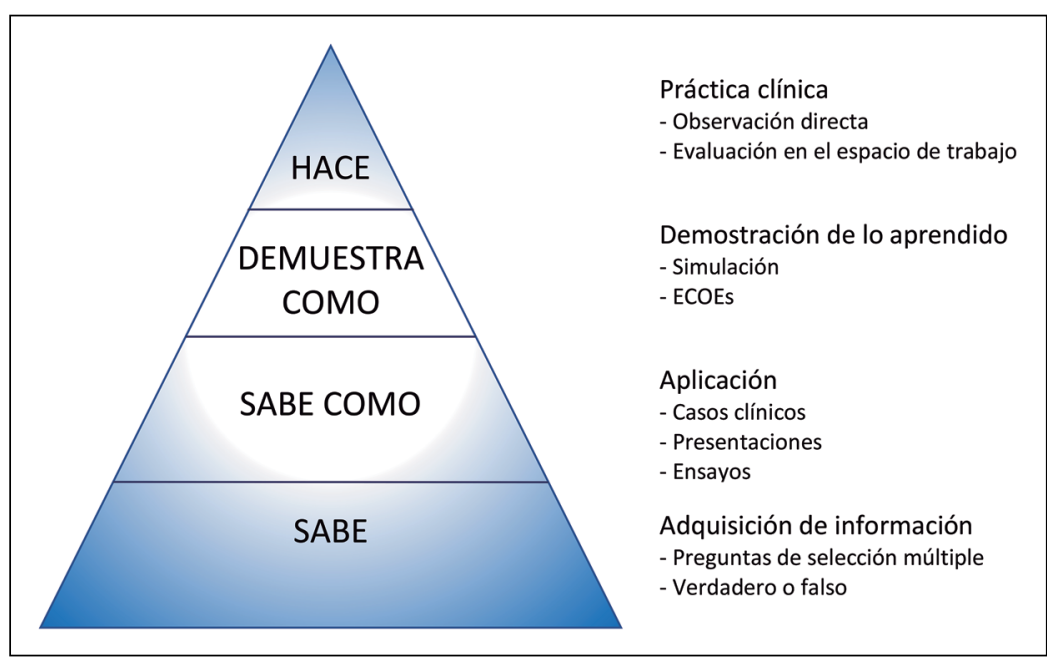

Figura 1. Pirámide de Miller junto con sus aplicaciones. 
La simulación también puede ser considerablemente costosa, particularmente si incluye modelos animales vivos o cadavéricos. Aparte de los simuladores, usualmente su implementación requiere un costo asociado de instalación y entrenamiento de personal para operarlos, lo cual puede dificultar su masificación en escuelas de medicina o programas de postgrado que no cuenten con los recursos ${ }^{24}$.

Consecuentemente, ha existido una proliferación de compañías privadas ofreciendo una variedad de cursos que cuentan con simulación. Aparte de la legítima motivación por perfeccionar habilidades clínicas, la competitividad por asegurar puestos de entrenamiento ha significado un aumento en la demanda por estos cursos que pudieran significar una ventaja competitiva para los candidatos ${ }^{25}$. Estas compañías con fines de lucro pudieran no ser siempre meticulosas en la formación académica entregada y existe un riesgo para los estudiantes que sus expectativas estén más basadas en publicidad y no en evidencia robusta.

Aun cuando la adquisición de competencias mediante simulación y su reproducibilidad en la práctica clínica ha sido reportada previamente en la literatura ${ }^{26}$, medir su real impacto en indicadores objetivos basados en resultados en pacientes es una pregunta de investigación más compleja ${ }^{27}$. A pesar del incremento exponencial en el número de artículos publicados relacionados con simulación, solo un número reducido se ha enfocado en esta disyuntiva. Una reciente revisión sistemática de artículos que midieron resultados en pacientes tras haber entrenado operadores mediante simulación, solo ha mostrado un pequeño a moderado beneficio. Agregando los resultados que compararon simulación versus ninguna intervención la diferencia de promedio estandarizada hacia un resultado favorable fue de $0,47(0,31-0,63)$ y los estudios que compararon simulación versus cualquier otro método de instrucción fue $0,36(-0,06-0,78)^{28,29}$. La gran disparidad en los distintos métodos de simulación y la heterogeneidad de métodos y resultados estudiados hacen que una revisión sea difícil de interpretar. Es por esta razón los autores de la mencionada síntesis proponen que hay una necesidad de estandarizar indicadores objetivos de manera de facilitar el análisis estadístico de la evidencia disponible. A pesar de esta situación, Issenberg ha reportado que ciertas características de simulación de alta fidelidad se asociaron en su experiencia consistentemente con un aprendizaje facilitado, incluyendo: proveer retroalimentación, permitir práctica deliberada, integración con el currículo, variabilidad en los escenarios simulados, apoyo du- rante el entrenamiento, aprendizaje individualizado y resultados esperados bien definidos ${ }^{30}$.

\section{Conclusión}

Incluso considerando sus limitaciones, la simulación moderna ha sido un gran avance para la educación médica. No solo por sus ventajas inherentes sino también considerando el contexto adverso que rodea la docencia clínica actualmente. Nuevas barreras a la educación médica han aparecido en un relativamente corto espacio de tiempo, haciendo a la simulación indispensable para nuestros días. Restricciones a las horas trabajadas, más conciencia en la seguridad de los pacientes, tasas de litigación médica sin precedentes y mayor demanda social por especialistas son desafíos muy distintos a los que enfrentaron los docentes durante el siglo XX.

Es así como muchos años después del redescubrimiento de la simulación como herramienta docente, el avance de nuevas tecnologías hápticas y de realidad virtual han significado un gran avance en la técnica. El incremento en las publicaciones relacionadas es un reflejo de este proceso, no solo justificando el desarrollo de mejores simuladores, sino también relacionando esta herramienta con diversas teorías educacionales como el conductivismo, cognitivismo, constructivismo social, aprendizaje reflexivo y práctica deliberada. Cada una de estas enriquece el concepto de simulación e inspira nuevas maneras de enseñar y evaluar a doctores en formación.

Es innegable que la simulación jamás podrá reemplazar el entrenamiento en la vida real. A pesar de que puede generar una falsa sensación de seguridad a los estudiantes que pueden no aun ser competentes, el problema ha sido identificado y hay estrategias para abordarlo. Retroalimentación de calidad parece ser la clave para mantener a los estudiantes motivados y reflexionando en su aprendizaje, a la vez que recordando sus diferencias con la práctica clínica.

Más interesante aún es la posibilidad que el uso de simulación ya parece demostrar una mejora en los resultados objetivos en el tratamiento de pacientes y no tan solo satisfacción de los estudiantes y docentes. Un esfuerzo en estandarizar estas medidas y disminuir la heterogeneidad de los estudios para aumentar la evidencia en la materia.

\section{Conflicto de intereses}

Los autores declaran no tener conflictos de interés. 


\section{Bibliografía}

1. Gholami-Kordkheili F, Wild V, Strech D. The impact of social media on medical professionalism: a systematic qualitative review of challenges and opportunities. J Med Internet Res [Internet]. 2013 Aug 28 [cited 2017 Mar 16];15(8):e184. Available from: http://www.ncbi.nlm.nih.gov/ pubmed/23985172

2. Kachalia A, Studdert DM. Professional Liability Issues in Graduate Medical Education. JAMA [Internet]. 2004 Sep 1 [cited 2017 Mar 16];292(9):1051. Available from: http://www.ncbi.nlm.nih. gov/pubmed/15339896

3. Klamen DL, Sanserino K, Skolnik P. Patient Safety Education: What Was, What Is, and What Will Be? Teach Learn Med [Internet]. 2013 Jan [cited 2017 Mar 16];25(sup1):S44-9. Available from: http://www.ncbi.nlm.nih.gov/ pubmed/24246106

4. Gupta A, Snyder A, Kachalia A, Flanders S, Saint S, Chopra V. Malpractice claims related to diagnostic errors in the hospital. BMJ Qual Saf [Internet]. 2017 Aug 9 [cited 2017 Nov 24];bmjqs-2017-006774. Available from: http://qualitysafety. bmj.com/lookup/doi/10.1136/ bmjqs-2017-006774

5. D'Souza N, Hashimoto DA, Gurusamy K, Aggarwal R. Comparative Outcomes of Resident $v s$ Attending Performed Surgery: A Systematic Review and Meta-Analysis. J Surg Educ [Internet]. 2016 May [cited 2017 Mar 16];73(3):391-9. Available from: http://www.ncbi.nlm.nih.gov/ pubmed/26966079

6. Hutter MM, Kellogg KC, Ferguson CM, Abbott WM, Warshaw AL. The impact of the 80-hour resident workweek on surgical residents and attending surgeons. Ann Surg [Internet]. 2006 Jun [cited 2017 Mar 18];243(6):864-71-5. Available from: http://www.ncbi.nlm.nih.gov/ pubmed/16772790

7. Hartle A, Gibb S, Goddard A. Can doctors be trained in a 48 hour working week? BMJ [Internet]. 2014 Dec 9 [cited 2017 Mar 1];349:g7323. Available from: http:// www.ncbi.nlm.nih.gov/pubmed/25498122

8. de Blacam C, Tierney S, Shelley O. Experience of plastic surgery registrars in a European Working Time Directive compliant rota. J Plast Surg Hand Surg
[Internet]. 2017 Jul 4 [cited 2017 Nov 24];51(4):264-9. Available from: http:// www.ncbi.nlm.nih.gov/pubmed/27762159

9. Torkington J, Smith SG, Rees BI, Darzi A. The role of simulation in surgical training. Ann R Coll Surg Engl [Internet]. 2000 Mar [cited 2017 Mar 17];82(2):88-94. Available from: http://www.ncbi.nlm.nih. gov/pubmed/10743423

10. Badash I, Burtt K, Solorzano CA, Carey $\mathrm{JN}$. Innovations in surgery simulation: a review of past, current and future techniques. Ann Transl Med [Internet]. 2016 Dec [cited 2017 Mar 17];4(23):453453. Available from: http://www.ncbi.nlm. nih.gov/pubmed/28090509

11. Lanzarini SE, Schonstedt PV, Abedrapo MM, Yarmuch GJ, Csendes JA, Rodriguez NA. Simulación: Una herramienta útil en la formación quirúrgica moderna. Rev Chil Cir [Internet]. 2008 Apr [cited 2017 Nov 24];60(2):167-9. Available from: http://www.scielo.cl/scielo. php?script=sci_arttext\&pid=S0718$40262008000200016 \& \operatorname{lng}=\mathrm{en} \& \mathrm{nrm}=\mathrm{iso} \&$ tlng=en

12. Corvetto M, Bravo MP, Montaña R, Utili F, Escudero E, Boza C, et al. Simulación en educación médica: una sinopsis. Rev Med Chile [Internet]. 2013 Jan [cited 2017 Nov 24];141(1):70-9. Available from: http://www.ncbi.nlm.nih.gov/ pubmed/23732417

13. Owen H. Early Use of Simulation in Medical Education. Simul Healthc J Soc Simul Healthc [Internet]. 2012 Apr [cited 2017 Mar 17];7(2):102-16. Available from: http://www.ncbi.nlm.nih.gov/ pubmed/22374231

14. Ker J, Bradley P. Simulation in Medical Education. In: Swanwick T, editor. Understanding Medical Education: Evidence, Theory and Practice. Second. Wiley-Blackwell; 2014. p. 175-92.

15. Maran NJ, Glavin RJ. Low- to highfidelity simulation - a continuum of medical education? Med Educ [Internet]. 2003 Nov [cited 2017 Mar 18];37 Suppl 1:22-8. Available from: http://www.ncbi. nlm.nih.gov/pubmed/14641635

16. Rehman AJ. A Handbook of Flight Simulation Fidelity Requirements for Human Factors Research [Internet]. Federal Aviation Administration Technical Center; 1995 [cited 2017 Mar 17]. Available from: https://
books.google.co.uk/books/about/A Handbook_of_Flight_Simulation_Fidelity. html?id=tyWwSgAACAAJ\&redir_esc $=y$

17. Malik MM, Hachach-Haram N, Tahir M, Al-Musabi M, Masud D, Mohanna P-N. Acquisition of basic microsurgery skills using home-based simulation training: A randomised control study. J Plast Reconstr Aesthetic Surg [Internet]. 2017 Apr [cited 2017 Mar 17];70(4):478-86. Available from: http://www.ncbi.nlm.nih. gov/pubmed/28161208

18. Konia M, Yao A. Simulation-a new educational paradigm? J Biomed Res [Internet]. 2013 Mar [cited 2017 Mar 18];27(2):75-80. Available from: http:// www.ncbi.nlm.nih.gov/pubmed/23554798

19. Schön DA. Educating the reflective practitioner: Toward a new design for teaching and learning in the professions. [Internet]. Jossey-Bass; 1987 [cited 2017 Mar 18]. Available from: http://psycnet. apa.org/index.cfm?fa $=$ search.displayRecor $\mathrm{d} \&$ uid $=1987-97655-000$

20. Ericsson KA. Deliberate practice and the acquisition and maintenance of expert performance in medicine and related domains. Acad Med [Internet]. 2004 Oct [cited 2017 Mar 18];79(10 Suppl):S70-81. Available from: http://www.ncbi.nlm.nih. gov/pubmed/15383395

21. Gladwell M. Outliers : the story of success [Internet]. First. Little, Brown and Company; 2008 [cited 2017 Mar 18]. 309 p. Available from: https://books.google. co.uk/books?hl=en\&lr $=\& \mathrm{id}=\mathrm{qQc7AQ}$ AAQBAJ\&oi $=$ fnd\&pg $=$ PT215\&ots $=8$ famjnOact\&sig=Y9UPxYmNCLfUrX7lv1CdnREg_s\&redir_esc $=y$

22. Miller GE. The assessment of clinical skills/competence/performance. Acad Med [Internet]. 1990 Sep [cited 2017 Mar 18];65(9 Suppl):S63-7. Available from: http://www.ncbi.nlm.nih.gov/ pubmed/2400509

23. Barnsley L, Lyon PM, Ralston SJ, Hibbert EJ, Cunningham I, Gordon FC, et al. Clinical skills in junior medical officers: a comparison of self-reported confidence and observed competence. Med Educ [Internet]. 2004 Apr [cited 2017 Mar 19];38(4):358-67. Available from: http://www.ncbi.nlm.nih.gov/ pubmed/15025637

24. Zendejas B, Wang AT, Brydges R, Hamstra SJ, Cook DA. Cost: The missing 


\section{ARTíCULO DE REVISIÓN}

outcome in simulation-based medical education research: A systematic review. Surgery [Internet]. $2013 \mathrm{Feb}$ [cited 2017 Mar 19];153(2):160-76. Available from: http://www.ncbi.nlm.nih.gov/ pubmed/22884087

25. Opel S, Ghani Y, Branford O. The insider's guide to obtaining a national training number in plastic surgery. Bull $\mathrm{R}$ Coll Surg Engl [Internet]. 2014 Oct [cited 2017 Mar 19];96(9):e1-5. Available from: http://publishing.rcseng.ac.uk/doi/10.1308 /147363514X14042954769474

26. Aebersold M, Tschannen D. Simulation in nursing practice: the impact on patient care. Online J Issues Nurs [Internet]. 2013
May 31 [cited 2017 Mar 19];18(2):6. Available from: http://www.ncbi.nlm.nih. gov/pubmed/23758424

27. Patel R, Dennick R. Simulation based teaching in interventional radiology training: is it effective? Clin Radiol [Internet]. 2017 Mar [cited 2017 Mar 19];72(3):266.e7-266.e14. Available from: http://www.ncbi.nlm.nih.gov/ pubmed/27986263

28. Zendejas B, Brydges R, Wang AT, Cook DA. Patient Outcomes in SimulationBased Medical Education: A Systematic Review. J Gen Intern Med [Internet]. 2013 Aug 18 [cited 2017 Mar 19];28(8):107889. Available from: http://www.ncbi.nlm. nih.gov/pubmed/23595919

29. Cook DA, Hatala R, Brydges R, Zendejas B, Szostek JH, Wang AT, et al. Technology-Enhanced Simulation for Health Professions Education. JAMA [Internet]. 2011 Sep 7 [cited 2017 Mar 19];306(9):978-88. Available from: http:// www.ncbi.nlm.nih.gov/pubmed/21900138

30. Issenberg SB, McGaghie WC, Petrusa ER, Lee Gordon D, Scalese RJ. Features and uses of high-fidelity medical simulations that lead to effective learning: a BEME systematic review. Med Teach [Internet]. 2005 Jan 3 [cited 2017 Mar 19];27(1):1028. Available from: http://www.ncbi.nlm. nih.gov/pubmed/16147767. 